\title{
The relation of lightness and stereoscopic depth in a simple viewing situation
}

\author{
TIMOTHY A. DALBY, MEREDITH L. SAILLANT, and B. R. WOOTEN \\ Brown University, Providence, Rhode Island
}

\begin{abstract}
The effect of stereoscopic depth on perceived lightness was studied using a simple, achromatic stimulus arrangement. In Experiment 1, depth/lightness interactions were sought between a single test field and a single induction field. In Experiment 2, depth/ightness interactions were looked for between a single test field and two induction fields. Stimuli were presented on a computer screen and viewed with a stereoscope. The subjects reported perceived lightness of the achromatic test field by rating its apparent blackness along a dimension of 0\%-100\%. In Experiment 1, they reported lightness judgments of the test field across 13 perceived depth levels and 8 contrast levels. In Experiment 2 , they gave lightness judgments of the test field across 7 perceived depth levels and 16 contrast levels. We were particularly interested in observing the generality of Gilchrist's coplanar ratio hypothesis. The results showed that when stereopsis and contrast levels are the available cues, depth and lightness percepts are independent, and it is retinal ratios, not coplanar ratios, that dictate lightness perception. We conclude that before the relative depth location of an object is determined, its lightness value is known through sensory-level processes.
\end{abstract}

Investigators have long been interested in determining at which level of processing lightness ${ }^{1}$ perception occurs. Broadly, two distinct classes of theories exist regarding this issue, one of which considers the perception of lightness to be a sensory-level process, while the other sees it as being processed at a higher cognitive level.

Sensory-level explanations of lightness are grounded in the research of Hering (1920/1964) and Wallach (1948). Hering proposed the theory of spatially opponent processes to explain lightness, whereby a light area of a surface has a blackening effect on the perception of an adjacent area of the surface. Support for this view came in 1948, when Wallach proposed the ratio principle, having concluded from his experiments that the luminance ratio between retinally adjacent regions determines the perceived achromatic color of a region. Here, the concept of contrast between two regions is key to the consideration of lightness. A particular shade of gray may only be perceived when the region of interest is seen in relation, or contrast, to another region of higher luminance. This view holds that low-level sensory mechanisms determine lightness.

Cognitive-level explanations are founded in a theory espoused by von Helmholtz (1867/1925) - that of unconscious inference. According to Rock (1983), "the perceptual system takes account of factors such as distance or illumination in arriving at a perceptual judg-

Correspondence concerning this article should be addressed to B. $R$. Wooten, W. S. Hunter Laboratory of Psychology, Brown University, Providence, RI 02912.

$$
\text { -Accepted by previous editor, Charles W. Eriksen }
$$

ment of what that retinal image represents in the world" (p. 241). This view holds that higher-level cognitive processes determine lightness.

One area in which proponents of these two approaches disagree, and which allows for their further examination, concerns the effect of perceived depth on lightness. While sensory approaches, per se, do not discount the possibility of a depth/lightness interaction, they view such an interaction as unexpected because lightness determination is seen as being the result of a sensory mechanism's response to luminance ratios. Some cognitive approaches, on the other hand, accommodate a depth/lightness interaction (Gilchrist, 1977), since different depth planes within a stimulus arrangement are seen as providing cues to illumination that are critical to lightness determination. It must be emphasized that although neither approach either denies or requires a depth/lightness interaction, an experimental finding of no interaction would support a sensory approach, while one which did find an interaction would support a more cognitive approach.

The effect of depth on lightness has been explored by a number of researchers attempting to determine whether lightness is best thought of as a sensory, contrast process or as a higher-level cognitive process. If lightness is determined at a stage prior to that of depth processing, depth between contrasting elements in an image should not influence lightness. However, if it is determined after stereopsis occurs (i.e., after the images from both eyes are combined in the visual cortex), depth between elements could be expected to influence lightness; that is, some cognitive process could then be determining lightness.

The results of experiments using fairly simple visualdisplay situations have conflicted with each other and remain inconsistent. Gogel and Mershon (1969; Mershon 
\& Gogel, 1970), using a Gelb-effect ${ }^{2}$ paradigm, claimed to find evidence of a large depth/lightness interaction. They found that as the separation in perceived depth increased between the low-reflectance disk and the highly luminous induction field, the low-reflectance disk appeared significantly lighter. The difference reported between the perceived lightness at zero depth and the most extreme depth was $39 \%$. Lie (1969) proposed that the large difference in lightness judgments reported by Gogel and Mershon (1969) were probably due to either lateraldisplacement effects, double images, or blurring. Instead of physical depth, Lie used a stereoscopic depth technique in an attempt to avoid these possible problems. Although Lie's results suggested a slight lightening of the test field as depth increased between test and induction fields, the mean results were not significant. Subsequently, Gibbs and Lawson (1974) used a stereoscopic presentation of a simultaneous-contrast display to study the effects of depth on brightness. Although they claimed to be studying brightness, their descriptions of their subjects' responses indicate that they were in fact studying lightness. They found a slight, but nonsignificant, effect of depth on lightness. However, this small effect was actually in the opposite direction to that predicted by Gogel and Mershon. As the test field moved out of the plane of the induction field, Gibbs and Lawson found that the contrast effect actually increased slightly. Finally, Julesz (1971) noted only a slight effect on perceived lightness when using random-dot stereograms to create depth in a simultaneous-contrast display.

In summary, the results of previous experiments conflict with each other regarding the effect of depth on lightness in a simple viewing situation. While some researchers (Gogel \& Mershon, 1969; Mershon \& Gogel, 1970) found a large effect, others (Gibbs \& Lawson, 1974; Lie, 1969) found either no effect or only a slight, nonsignificant one. Thus, the level at which lightness processing occurs remains unclear. If lightness is affected by stereoscopic depth, it remains possible that cognitive processes determine lightness perception, but if there is no effect of depth on lightness, a sensory-level explanation is clearly supported.

Although some previous research (Gilchrist, 1977, 1980; Mershon, 1972; Schirillo, Reeves, \& Arend, 1990) found support for depth effects on lightness in more complex viewing situations, possible problems remain. Complex viewing situations often involve stimulus displays with multiple induction fields of differing areas and luminance levels. The difficulty of interpreting results from such displays stems largely from the unknown lightness interactions that occur between the many induction fields and, hence, the unknown impact that such interactions have on the test field. Results of experiments using natural viewing situations may also have been affected by uncontrolled aspects of illumination, such as shadows, edge discontinuities, specular reflection, and other inhomogeneities.

Mershon (1972) was the first to examine the issue using a complex viewing situation containing a single test field and two induction fields. The two induction fields were separated in depth and laterally displaced from one another. Additionally, they were both divided into two halves, such that one half appeared vertically above the other half, from which it was separated by a gap equal to the width of each half. The test field was given abundant space to appear within the gap between these two halves. The entire display, then, contained five fields of varying luminance values all separated from one another by varying degrees of a low-luminance background. The results of this study are particularly difficult to interpret because of the unknown interactions between the many isolated induction fields, the test field, and the gaps in the display.

Though also complex, research by Gilchrist (1977, 1980) led to his development of a new hypothesis for lightness perception-the coplanar ratio hypothesis. This states that the ratio that determines perceived lightness is "the ratio between regions that are perceived to lie next to one another in the same spatial plane" (Gilchrist, 1980, p. 530). Gilchrist found that when subjects were shown a contrast display, if depth cues were available such that the target stimulus appeared in the plane of one comparison field rather than in that of another, these cues apparently affected lightness judgments. Gilchrist (1980) concluded that the results of previous studies, in which lightness was found mostly to be invariant of perceived depth, may have been affected by a "default outcome" (p. 533), such that lightness was determined by the luminance ratio between retinally adjacent regions.

Although Gilchrist (1980) supplied much evidence to support his coplanar hypothesis, the complex nature of the visual display presented to subjects makes interpreting his results more difficult. In addition to the problems already mentioned involved in natural viewing situations - particularly those involving the juxtaposition of multiple induction fields- the task of matching for lightness or brightness in such a paradigm may also become confused. In fact, in Gilchrist's $(1977,1980)$ studies, the subjects were never informed of the dimensions of lightness and brightness when making perceptual judgments, nor were they asked to distinguish between them.

Recently, in an attempt to gain more control over the stimulus setting, Schirillo et al. (1990) tested Gilchrist's $(1977,1980)$ theory using a computer-screen presentation of his complex viewing situation. Although their results were in the same direction as those of Gilchrist, they were smaller in magnitude, and their interpretation remains problematic due to the nature of the response measure, whereby the subjects were either to select from a grid of comparison patches or to vary the intensity of a comparison patch, such that the patch "look[ed] as if it were cut from the same piece of paper" as the test patch (Schirillo et al., p. 90). Under these conditions, they were required to make a series of inferences about the stimulus on the screen before they reported each lightness judgment: First, they had to imagine that the test patch on the screen was made of paper; next, they had to 
choose the correct "paper" to match this, either from the grid or from the variable comparison field. It is the high level of cognitive processing necessary to complete this task, above and beyond the processing needed to report the lightness judgment, that makes these results more difficult to interpret.

This paper reports the findings of two sets of experiments investigating depth/lightness interactions in simple viewing situations. The first set (Experi nent 1) was designed to clarify the inconsistent findings of previous studies involving a single induction region and a single test region (Gibbs \& Lawson, 1974; Gogel \& Mershon, 1969; Lie, 1969; Mershon \& Gogel, 1970). The second set (Experiment 2) was aimed at investigating further the viewing situations previously studied by Gilchrist ( 1977 , 1980), Mershon (1972), and Schirillo et al. (1990)namely, those involving multiple induction fields and a single test field-in order to test Gilchrist's coplanar ratio hypothesis.

To avoid the display complexities and interaction problems encountered in previous research, the present study used very simple viewing arrangements. In Experiment 1 , a square surround of middle-level luminance, with a square center test region of variable luminance, was set against the lowest obtainable CRT luminance. A pair of such stimuli were presented on a computer screen and were viewed with a stereoscope. This arrangement allowed very fine disparity settings and assured crisp edges, homogeneous luminosities, and no possibility of shadows. In Experiment 2, the conditions were similar, except that two induction fields were used rather than only one.

The present study also examined the entire stimulus space of perceived depth and lightness, in contrast to earlier studies, which had sampled from only a few small pockets of this space. In Experiment 1, the full range of perceived lightness was tested, from black, through shades of gray, to white, by using 8 contrast ratios. ${ }^{3}$ Previous studies involving one test and one induction field had used a maximum of 3 contrast ratios (Lie, 1969). Our study, by using 13 levels of disparity, also tested the full range of perceived depth, such that the extremes of fusion were reached. Earlier research (Gibbs \& Lawson, 1974; Lie, 1969) had used only 5 depth levels. By using this wider range of contrast and disparity levels, it was possible in the present study to examine Mershon's (1971) suggestion that the effect of depth on lightness would be facilitated by strong contrast effects.

In Experiment 2, the full range of perceived lightness was again tested, using a total of 16 contrast ratios between the test and induction fields (whereas previous multiple induction field studies had used only 3 [Mershon, 1972; Schirillo et al., 1990]), and seven depth levels (compared with 5 at the most in earlier studies [Mershon, 1972]).

As a further improvement upon previous research, the subjects in the present study were asked to report perceived lightness using an absolute-judgment paradigm; that is, lightness judgments of the test region were reported in terms of the percentage of blackness perceived (ranging from $0 \%$ to $100 \%$ ). This was based upon the research of Quinn, Wooten, and Ludman (1985), who had shown that the dimension of lightness may be completely described using the color terms black and white. This method actually informed the experimenters of subjects' perceptual experiences and thus allowed the size of any lightness changes to be evaluated. Further, possible problems associated with matching were avoided, such as those caused by subjects needing to shift their gaze from the pattern to be fused to a comparison field (Gilchrist, 1977, 1980; Schirillo et al., 1990). Although not utilizing this technique in his own study, Mershon (1972) remarked that a procedure "[such] as naming may provide precise and reliable information about appearance" (p. 970).

\section{EXPERIMENT 1}

\section{Method}

\section{Subjects}

Three males and six females, whose ages ranged from 23 to 29 years, served as subjects in all trials. All but one were graduate students at Brown University, and all were naive concerning the purpose of the study.

\section{Apparatus}

Stereograms varying in contrast ratio and disparity were displayed on an Apple color, high-resolution RGB monitor with a surface measuring $32.4 \mathrm{~cm}$ diagonally. The stimuli were displayed using MacLaboratory's Reaction Time program (Version 2.0) with an Apple Macintosh II computer. The stereograms were viewed through a flat black viewing tube measuring $100 \times 26 \times 20 \mathrm{~cm}$ (length $\times$ width $\times$ height). A septum was positioned in the tube such that each eye received only one image of the stereogram pair. The subjects fused the pair of images with the aid of four diopter prisms positioned before each eye. A chin rest and a forehead rest maintained each subject's head position for correct viewing.

\section{Stimuli}

Main experiment. The stereogram stimuli were created on the Apple Macintosh II computer using Pixel Paint (Version 2.0) software from SuperMac. The stereograms were centrally positioned on the computer screen against a low-luminance background $(0.014 \mathrm{fL}$, as measured by a Spectra Pritchard photometer $)$. The square surround regions measured $4.8 \times 4.8 \mathrm{~cm}$ and had luminance values of $6.93 \mathrm{fL}$ in all but one case, the exception requiring a setting of $7.81 \mathrm{fL}$ to attain a desired contrast ratio. The center square regions measured $1.86 \times 1.86 \mathrm{~cm}$ and varied in luminance across 8 values. The resulting luminance ratios between the centers and surrounds were $0.01,0.03,0.09,0.18,0.29,0.43,0.84$, and 1.43. Stereoscopic depth, using retinal disparity, was induced by presenting a center/surround stimulus pattern to each eye with the center test square laterally displaced (see Figure la). Center regions varied in disparity with respect to the surround regions across 13 levels. The disparity levels, in minutes of arc, were: \pm 60 , $\pm 46, \pm 30, \pm 20, \pm 10, \pm 4$, and 0 . The center-to-center distance used to attain optimal fusion was $7.9 \mathrm{~cm}$. The visual angles of the center test region and the surround region were $1.14^{\circ}$ and $2.86^{\circ}$, respectively.

As small changes in contrast have relatively large effects on lightness, contrast ratios needed to be stable and accurate throughout the experiment. This required the various regions of the stim- 


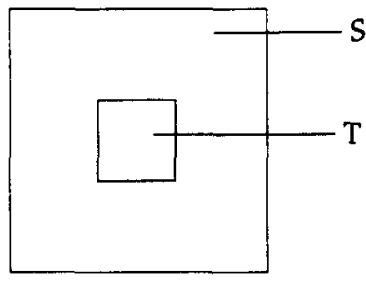

a

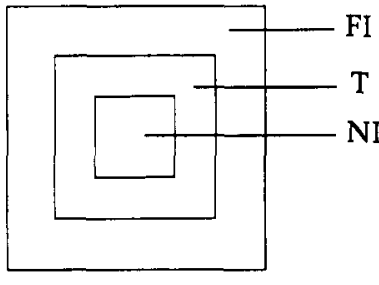

b
Figure 1. (a) Stimulus used in Experiment 1 ( $T=$ test stimulus; $S=$ surround). (b) Stimulus used in Experiment 2 ( $T=$ test stimulus; $N I$ = near inducer; $\mathbf{F I}=$ far inducer).

ulus (i.e., center, surround, and background) to be homogeneous throughout, such that contrast ratios remained constant at all points along the boundary between each region. To determine the accuracy of the contrast ratios used, an image of the stimuli presented on the computer monitor was formed in the plane of a United Detector Technologies photodiode (Model 111A). This was accomplished by using two lenses, the first at one focal length from the computer screen, and the second at one focal length from the photodiode. Stray light was eliminated by passing the projected light through a series of tubes and baffles. This technique allowed us to determine that the relative luminances of the center and surround regions were constant at each contrast ratio and, having done so, to check that there was nothing lacking in homogeneity near or at the boundary between the two regions. This check was performed on those stimuli in which the test region appeared in the center position and on those in which it appeared in the most extreme laterally displaced positions. Luminance values on each side of the center/surround boundary were sampled using a Spectra Pritchard photometer with a 2 ' aperture stop. Four measurements were taken at or near each center/surround boundary, two each at 2 ' intervals on either side of the boundary. Only a slight variation in luminance (less than $4 \%$ ) was detected between the sampled points. This minor variation in contrast near the edges had no effect on the subjects' responses to the stimuli, as is evident from the results of perceived lightness at each contrast ratio in Figure 2 (discussed below).

Control experiment. A second set of stereograms was created and presented in the same way as those of the main experiment. As described above, the lateral displacement of the test region was a necessary condition for the induction of stereoscopic depth. However, because this displacement required a change in the stimulus pattern presented to each subject, this change in and of itself may have contributed to the perceived lightness. Thus, the control experiment was designed to test for an effect of the lateral displacement of the center region on lightness judgments. This was accomplished by presenting to each eye identical images of the center square's position against the surround square. The center region appeared to each eye in three locations for each contrast ratio-in the extreme left position, in the extreme right position, and in the center position. Thus, no depth effect occurred, but the center square appeared in positions that were analogous to the $-60^{\prime}$, $+60^{\prime}$, and $0^{\prime}$ disparities of the main experiment.

\section{Design}

Main experiment. The subjects viewed all 8 contrast ratios at all 13 disparity levels. Thus, each subject was presented with 104 different stimuli, which constituted a block of trials. Each block was presented eight times, for a total of 832 lightness judgments per subject.

Control experiment. As a control, all eight contrast ratios were presented across the three positions of the center square. This block of 24 trials was presented eight times to each subject, for a total of 192 lightness judgments per subject.

\section{Procedure}

All experiments were performed in a room illuminated by only the small amount of light escaping from the baffled computer screen. Before each experimental session, the subject's interpupilary distance was measured, and the prisms of the stereoscope were adjusted accordingly. The chin rest was also adjusted so that the subject's head was correctly aligned vertically. The United Detector Technologies photometer was used at this time to check the luminance output of the computer screen as it presented a middlelevel luminance adaptation field $(8.0 \mathrm{fL})$. Any deviation from the appropriate output level was corrected by adjusting the luminance with the brightness control on the computer screen.

Main experiment. The main experiment consisted of two tasks. Task 1 , the introductory experiment, tested for a depth percept, in order to verify that in our paradigm, with the particular apparatus and stimuli used, a reliable and expected effect of depth would indeed occur. It took a single 1 -h session to complete. Task 2 tested for a depth/lightness interaction, and consisted of four $1 \frac{1}{2}-\mathrm{h}$ sessions.

In Task 1, the subjects were presented with stimuli that sampled the range of contrast ratios and disparity levels; that is, they were shown stimuli with contrast ratios $0.01,0.29$, and 1.43 across disparity levels $\pm 60^{\prime}, \pm 20^{\prime}, \pm 4^{\prime}$, and $0^{\prime}$. The 21 stimuli were randomly presented to each subject twice. The subjects reported perceived depth (in inches) and direction (i.e., "in front" or "behind") of the center in relation to the surround region.

Task 2 of the main experiment consisted of the random presentation of all 104 experimental stimuli in a block, eight times. Two blocks were shown to each subject in approximately $11 / 2 \mathrm{~h}$. The subjects took breaks whenever necessary. They were initially read the following instructions:

You will be shown a series of images on the computer screen. These images will consist of a smaller square region within a larger square region. I will ask you to make some judgments about the center square region. Always ignore the surround. That is, make your judgments only about the center region.

You will view the images through a viewing tube and a set of prisms. The images have been designed such that sometimes the smaller square appears in front of the larger square, sometimes it appears behind the larger square, and sometimes it appears in the same plane as the larger square.

Each center square will contain some amount of whiteness and some amount of blackness. I would like you to judge the percent blackness that appears in the center square. For example, if the square region appears entirely white with no amount of black, that would be $0 \%$ black. The reverse could also be true, that is, the square could appear $100 \%$ black. Any intermediate value is also possible; a gray that is equally black and white would be $50 \%$ black. A gray that is 1/4 black would be $25 \%$ black. After the stimulus goes off, tell me your judgment of the percent blackness of the center square region. Give this judgment as quickly as possible after the stimulus has disappeared. Do you have any questions? (Adapted from Sewall, 1990)

The instructions were the same for each depth-experiment session, but were modified appropriately for the control experiment.

Control experiment. The 192 presentations of the control experiment took approximately $1 \frac{1}{2} \mathrm{~h}$, and were completed in a single session.

Main and control experiments. For all experiments, the spatial and luminance quantities of the stimuli, as noted above, all remained constant, except for the independent variables of contrast and disparity. The temporal aspect, which also remained constant, and the luminance quantities of the adapting fields were as follows. At all times other than the running time of the experiment, the subjects saw an 8.0 -fL adapting field. As a trial began, three successive adapting fields appeared, one at $38.0 \mathrm{fL}$ for $1.4 \mathrm{sec}$, one at $8.0 \mathrm{fL}$ 
for $1.5 \mathrm{sec}$, and one at $38.0 \mathrm{fL}$ for $1.3 \mathrm{sec}$. The stimulus field then appeared for $6 \mathrm{sec}$, and was followed by the 8.0 -fL adapting field for $1.5 \mathrm{sec}$, and then by the $38.0 \mathrm{fL}$ adapting field for $2.2 \mathrm{sec}$. Finally, an 8.0-fL adapting field reappeared during the intertrial interval (ITI), which lasted approximately $8 \mathrm{sec}$. The total running time for each trial was $13.9 \mathrm{sec}$. Ideally, the experiment would have been conducted without the presentations of the 38.0 -fL adapting field, which had to be made because of some restrictions of the software used to control stimuli presentation. The contrast ratios of the stimuli, however, remained constant throughout their display time, despite the restrictions noted previously.

\section{Results and Discussion}

Figure 2 shows the mean responses for the 8 contrast ratios at zero disparity for all nine subjects. It is clear from this figure that the subjects perceived the center square as very black (near $100 \%$ black) in the case of a low center luminance in relation to the surround's higher luminance. As the luminance of the center increased and approached that of the surround, perceived blackness decreased. Sewall (1990) found that as contrast between center and surround approached and exceeded a 1:1 ratio, perceived blackness remained about $5 \%$ on average. The inflection located at the 1.0 contrast position in Figure 2 reflects this finding. Figure 2 also shows that when the luminance of the center was higher than that of the surround (termed a high-contrast ratio), the center appeared very light (about $5 \%$ black), and contrast ratios in the middle range appeared as various shades of gray. The shape of the curve shown here is almost identical to the percentage blackness as a function of contrast plotted by Sewall, using a Maxwellian-view optical system.
Figure 2 also displays the results of the control experiment. As already discussed, in the images presented to both eyes, the lateral positions of the center test regions (i.e., extreme left, extreme right, and center), were displaced equally and in the same direction. Thus, a monocular change occurred for the position of the center test region without a change in depth occurring. The control was performed to verify that any effect on lightness that occurred in this viewing situation was not simply due to a positional change of the center region. As the three functions for the three positions may be seen to lie on top of one another, it is clear that lateral displacement had no effect on perceived lightness, and that contrast level was the sole determinant of lightness. This point is especially apparent when it is noticed that the zero-disparity function from the main experiment and the three functions from the control data are virtually indistinguishable.

Figure 3 shows mean responses for perceived depth during the introductory experiment, for 3 of the 8 contrast ratios and 7 of the 13 disparity levels. The ratios chosen represent the two most extreme cases (in which the centers appeared either predominantly black or predominantly white) and one ratio that appeared gray. It is clear from this figure that perceived depth increased as a function of degree of disparity. More extreme disparities, regardless of sign (i.e., whether positive or negative), appeared farther in depth from the plane of the surround region than did less extreme disparities. As expected, positively disparate stimuli appeared in front of the inducing field, while negatively disparate stimuli appeared behind the inducing field.

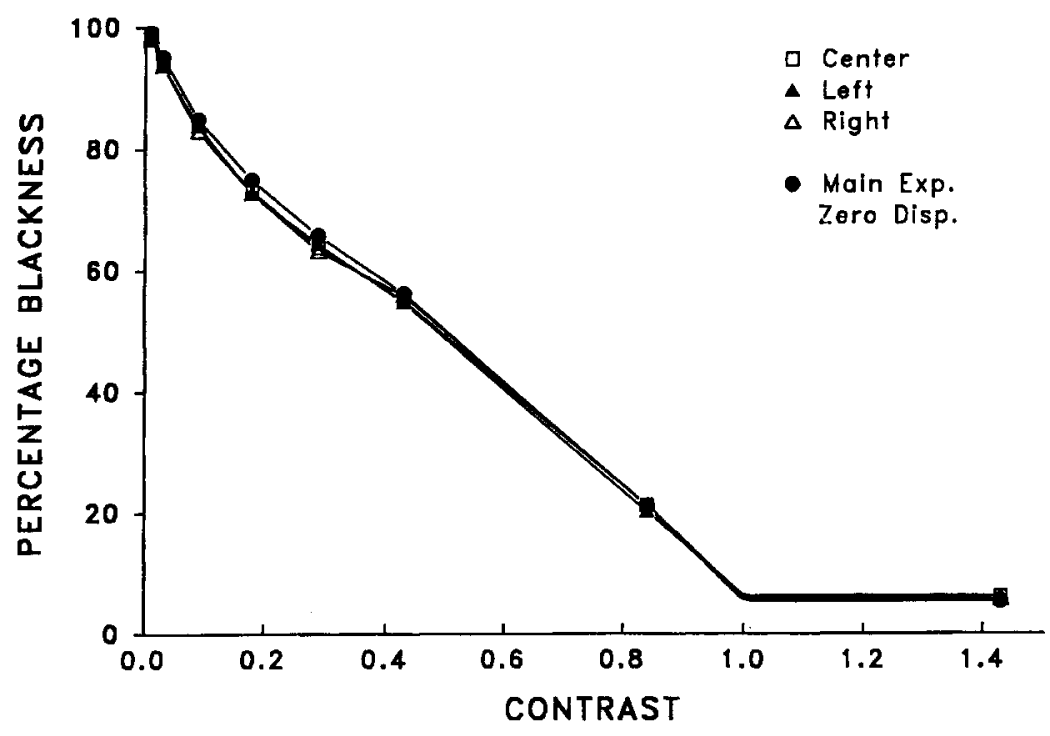

Figure 2. Main experiment: mean percentage of blackness plotted as a function of contrast ratio, at zero disparity. Also shown are the mean data for the control experiment at the three lateral positions of the test square. The four functions are almost identical. The data are averages for all nine subjects. Low contrast ratios elicited high percentage-blackness responses, while high contrast ratios elicited responses of around $5 \%$ blackness. Subjects reported various shades of gray for contrast ratios in the middle range. See text for rationale regarding inflection point at 1.0 contrast position. 


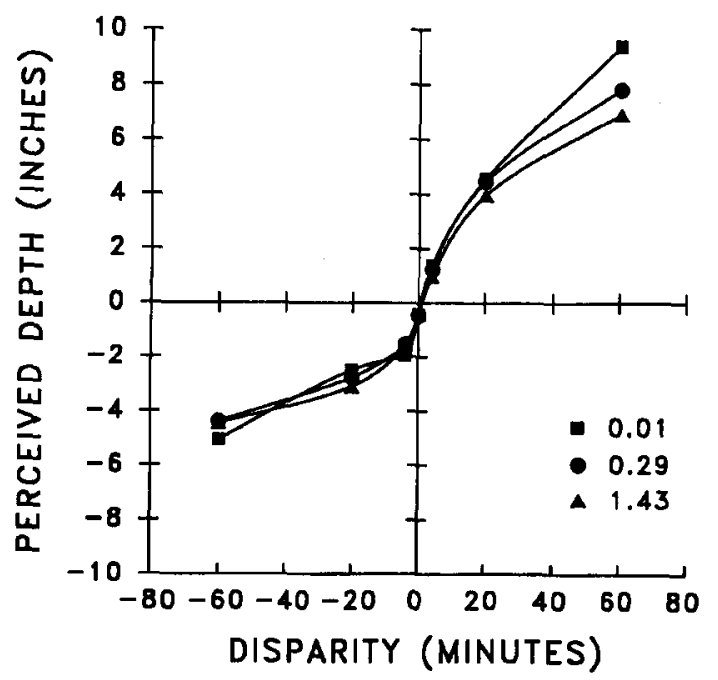

Figure 3. Mean perceived depth between test and induction fields as a function of disparity. The data are averages for all subjects. The figure legend indicates the three contrast conditions tested. Perceived depth increased as a function of increasing disparity, regardless of sign. Stimuli with more extreme disparity values appeared farther from the surround region than did stimuli of less extreme disparity.

Figure 4 plots the mean responses regarding percentage of blackness as a function of disparity for all 8 contrast ratios. Two regression lines were plotted at each contrast level, one through the positive disparities, and one through the negative disparities. This was done to test the two alternative positions that have been proposed in the literature - namely, (1) that lightness increases as the test field is moved away from the inducing field in depth (Gogel \& Mershon, 1969), and (2) that there is no effect on lightness when depth is changed (Gibbs \& Law- son, 1974; Lie, 1969). If the first view is correct, and depth affects lightness by decreasing the amount of blackness induced, we should expect to see lines that have positive slopes for negative disparities and negative slopes for positive disparities. Out of the 8 contrast levels, however, only $2(0.18$ and 1.43$)$ showed this positive-slopenegative-slope combination. Given the four possible combinations of positive and negative slopes for the 8 contrast levels, two such combinations would be expected by chance alone. Furthermore, a visual examination of these functions reveals that all of the lines have approximately zero slope; in fact, the greatest slope found was $0.03 \%$ blackness $/ \mathrm{min}$ of arc at the 0.09 contrast ratio.

Figure 5 (panels A and B) offers further support for the alternative view - namely, that depth has no effect on lightness. This figure displays the magnitude of the slopes of regression lines plotted for positive and negative disparities as a function of contrast, for each of the nine subjects. The slopes for each individual hover around the zero point, with no apparent order regarding the type of disparity; that is, the slope of lines at any particular contrast ratio or disparity level do not greatly favor either positive or negative slopes. This becomes especially apparent from the average slopes in the bottom pair of functions in panel B.

An effect, if one exists at all, would seem to be only very slight in Experiment 1, as the regression lines for positive and negative disparities are virtually flat. However, because they do not lie absolutely flat, the possibility remains that depth had some effect on lightness. Therefore, the data were subjected to a chi-square goodnessof-fit test. The slopes of the regression lines at contrast ratios of $0.03,0.09,0.18,0.29,0.43$, and 0.84 were considered for each individual. The two extreme contrast levels $(0.01$ and 1.43$)$ were excluded to avoid possible

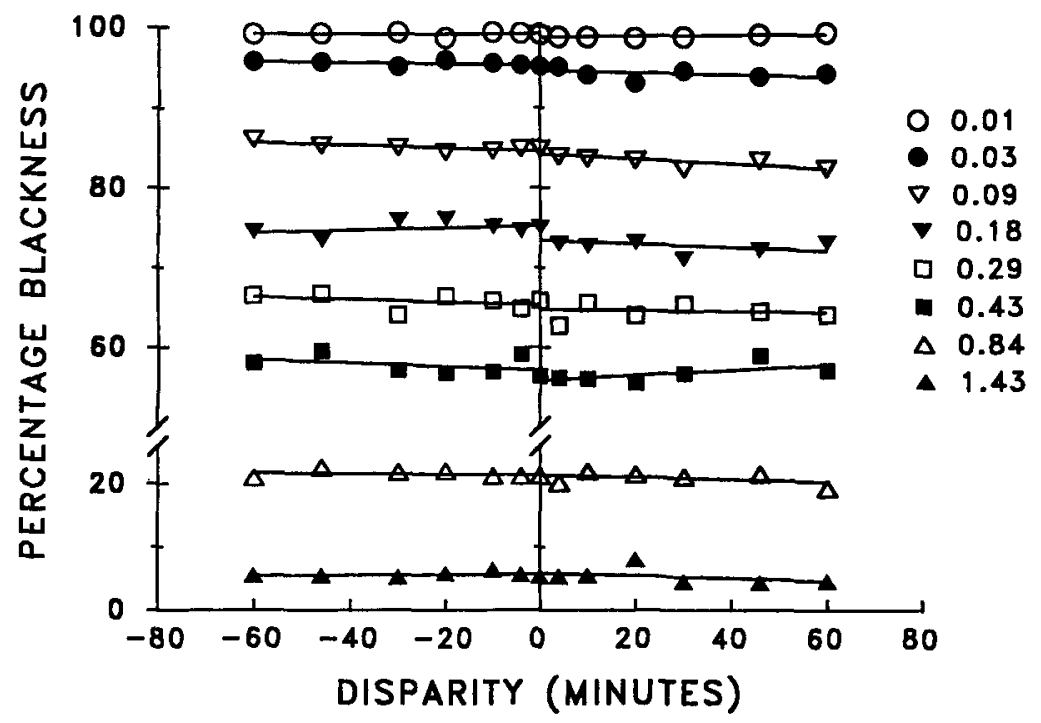

Figure 4. Percentage of blackness as a function of disparity. Two regression lines intersect the data points of each contrast condition, one for positive disparities and the other for negative disparities. All functions approximate zero slope. The data are averages for all subjects. 

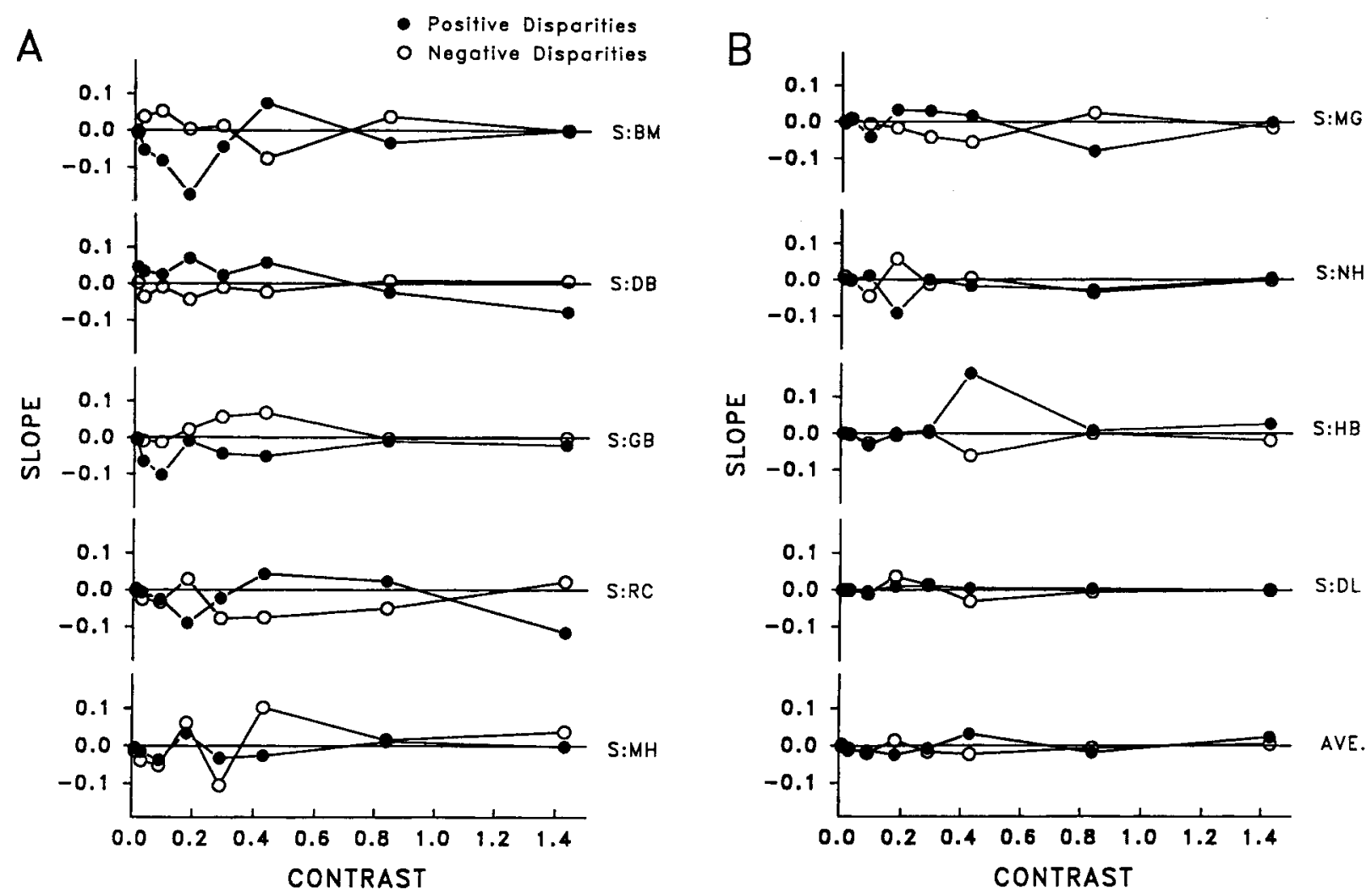

Figure 5. Slope of regression lines as a function of contrast condition. Slopes for each subject at each contrast condition are shown in panels $A$ and $B$. The average slopes for all nine subjects appear in the lowest plot of panel $B$.

floor and ceiling effects, and to provide a more conservative test and thus make it more likely that any significant effects would be found, if they existed. When the chi-square goodness-of-fit test was computed, no effect of depth on lightness was found $\left[\chi^{2}(3, N=54)=6.15\right.$, $p>.1]$.

The results of Experiment 1 strongly suggest that lightness is independent of perceived depth in a simple viewing situation and, as such, confirm the findings of Gibbs and Lawson (1974) and Lie (1969) using a simpler display, and extending over a much larger range of disparities and contrast ratios. The present study effectively explored the whole stimulus space. Additionally, it avoided the problems of natural viewing by using a computer display and, by assuring that subjects could fuse images at the required distance with appropriate prisms, also avoided the criticism that Mershon (1971) leveled at Lie's research in this regard.

These results, however, contradict those of Gogel and Mershon (1969), who found that "[the lightness] induction occurring between two objects of different luminance is a decreasing function of their separation in stereoscopic depth" (p. 16). The present study revealed no function, linear or otherwise, to support this claim. As mentioned previously, the most extreme mean slope found was $0.03 \%$ blackness $/ \mathrm{min}$ of arc for positive disparities at the 0.09 contrast ratio (see Figure 4). Thus, the percentage of blackness perceived for this extreme example changed by $1.8 \%$ across $60^{\prime}$ of disparity. Gogel and Mershon reported a 39\% change in lightness when the induction field moved in depth from 0 to $34.2^{\prime}$ of disparity. We calculate that the lightness-induction effects they reported would be equal to a $7.2 \%$ blackness change in terms of the response measure used in Experiment 1. Thus, their rate of lightness-induction decrease would be about $0.21 \%$ blackness $/$ min of arc - or about seven times the size of the effect found in our experiment for the most extreme slope. Probable explanations for their results, especially regarding potential methodological problems, will be further considered in the general discussion section.

Despite the results of Experiment 1, however, the possibility remains that the perceptual system treats retinally adjacent ratios in a default outcome, as described by Gilchrist (1980). Experiment 2 evaluated Gilchrist's coplanar ratio hypothesis using the most simple stimulus arrangement possible one, that is, in which a test field and two induction fields are presented stereoscopically-in conjunction with the absolute-judgment paradigm. 


\section{EXPERIMENT 2}

\section{Method}

\section{Subjects}

One male and six female undergraduates at Brown University served as subjects. All had normal or corrected-to-normal vision and were screened for depth perception with a Keystone Televiewer stereoscope before the first testing session. Each subject was naive, and was able to perceive depth to at least $1^{\prime}$ of arc.

\section{Apparatus}

The apparatus was the same as that employed in Experiment 1.

\section{Stimuli \\ The stimuli were produced on an Apple Macintosh II computer using Canvas (Version 3.0) software.}

Main and test experiments. For each subject, each eye was presented with an image consisting of three differently sized square regions (see Figure 1 b). A small square region $(2.16 \times 2.16 \mathrm{~cm}$ and $1.2^{\circ}$ of visual angle) appeared within a larger square region $\left(3.8 \times 3.8 \mathrm{~cm}\right.$ and $\left.2.15^{\circ}\right)$, which in turn appeared within an even larger square region $\left(5.38 \times 5.38 \mathrm{~cm}\right.$ and $\left.3.03^{\circ}\right)$. Thus, for the zero-disparity condition, each monocular image appeared as a $1.2^{\circ}$ square contiguous with a square surround that was $.475^{\circ}$ thick, which, in turn, was contiguous with a second square surround that was $.44^{\circ}$ thick. The small square region and the largest surrounding square region were both treated as induction fields, while the middle region was the test field. All appeared against a low-level luminance background $(0.014 \mathrm{fL})$. The far induction field (the largest region) served as the frame of reference, and there was therefore no disparity between the images of this object presented to either eye. The small induction square was created with a crossed disparity of $34^{\prime}$ of arc with reference to the outer surround. This disparity made the region appear to lie in a plane that was considerably nearer to the subject than the outer region was. Hence, we refer to the small square field as the near inducer and to the outer surround as the far inducer. The middle test region varied in crossed disparity with respect to the outer induction region across 7 levels, from 0 to $34^{\prime}$. The disparity levels in minutes of arc were $0,6,13,20,27,31$, and 34 . The middle test region therefore appeared to be either in the same perceptual plane as the outer region $\left(0^{\prime}\right.$ of arc), in the same perceptual plane as the nearer region ( $34^{\prime}$ of arc), or at some point in between. Luminance levels of the far and near regions were produced according to one of the following two conditions: (1) the hi/lo condition, with the far inducer at $5.15 \mathrm{fL}$ and the near inducer at $4 \mathrm{fL}$, as tested by a Spectra Pritchard photometer; or (2) the lo/hi condition, with the far inducer at $4 \mathrm{fL}$ and the near inducer at $5.15 \mathrm{fL}$. The luminance level of the test region varied for each stimulus, producing contrast ratios of $0.01,0.06,0.15,0.28,0.45,0.61,0.81$, and 1.28 relative to the induction field of high luminance, and ratios of $0.01,0.08$, $0.19,0.36,0.58,0.79,1.04$, and 1.28 relative to the induction field of low luminance. The contrast ratio of 1.28 involving the field of higher luminance was obtained by lowering the luminance of this inducer by $5 \%$, to $4.95 \mathrm{fL}$.

Control experiment. Four types of control stereograms were presented to each of the subjects. All stimuli presented involved no perceived depth, and were therefore identical for each eye. Each of the four types of stimuli were presented in both hi/lo and lo/hi conditions, and at all contrast ratios. Type 1 and Type 2 stereograms were designed to test for an effect of the lateral displacement of the near induction field on lightness judgments. In Type 1 stereograms, the test field was centered against the far induction field, while the near induction field was aligned with the rightmost edge of the test region. In Type 2 stereograms, the near induction region was centered against the test region, which was in turn centered against the far induction field. This stereogram type also served to obtain baseline lightness judgments of the test field with both induction regions present. Stereogram Types 3 and 4 were designed to test for the effect of contrast ratio on lightness judgments when the test field appeared either with only the far induction field (Type 3 ) or with only the near induction field (Type 4). For both stimulus types, test and induction regions were both centered with respect to each other.

\section{Design}

Main experiment. The subjects viewed all possible combinations of disparity level, contrast ratio, and hi/lo and lo/hi conditions. Thus, each subject was presented with 112 different stimuli, and as each stimulus was randomly presented six times, a total of 672 lightness judgments were made by each subject.

Control experiment. As a control, the subjects viewed all combinations of stimulus type, contrast ratio, and hi/lo and lo/hi conditions (64 in all) six times in a random order. Two stimuli (consisting of the test and the near induction regions, in both hi/lo and lo/hi conditions, at the 0.01 contrast ratio) were eliminated because, at this level, the test region blended with the low-level luminance background and made it appear as if there were only one field on the screen. With these stimuli removed, therefore, a total of 372 lightness judgments were made by each subject.

\section{Procedure}

The same procedure was followed as in Experiment 1. The subjects were instructed to ignore the induction fields and to make their lightness judgments only about the test region.

Main experiment. This consisted of two tasks. Task 1 was a practice session, or test experiment, the goals of which were: (1) to expose the subjects to the types of stimuli with which they would be presented in the main experiment; (2) to familiarize the subjects with the task of judging the lightness of the stimuli; and (3) to test the subjects' depth perception for a second time. Stimuli from every disparity level and every combination of luminance level were shown in a randomized order. Task 2 , the main experiment proper, tested for a depth/lightness interaction.

Task 1 was completed during a single 30 -min session that included two types of judgments-namely, (1) a rating of percentage of blackness of the test region, and (2) a rating of the spatial position of the test field relative to the two induction fields. The spatialposition rating was given on a scale of 0 -10, where the test field was judged to be at Position 0 if it lay in the same plane as the far induction field, and at Position 10 if judged to be in the same plane as the near induction field. The test could lie anywhere in between these two extremes; if it were judged to be, for example, at a point halfway between the two induction planes, its rating would be 5 .

Task 2, the main experiment, consisted of the random presentation of all 112 stimuli, each of which was presented six times over the course of seven 30 -min sessions.

Control experiment. The 372 presentations of the control experiment were completed in four 30 -min sessions.

Main and control experiments. For all experimental sessions, at all times other than when they were presented with a stimulus, the subjects saw a homogeneous, low-level luminance screen $(0.014 \mathrm{fL})$. The ITI for each trial was $12 \mathrm{sec}$. A warning beep sounded $2 \mathrm{sec}$ before the stimulus was presented. The stimulus then appeared for $6 \mathrm{sec}$ before the low-level luminance screen reappeared.

\section{Results and Discussion}

Results of the test experiment and follow-up sessions indicated that all of the subjects perceived depth with the apparatus as expected. Using the $0-10$ scale, they were able reliably to rate stimuli either as being coplanar with the near or far induction fields, or as lying at specifiable locations in between. 


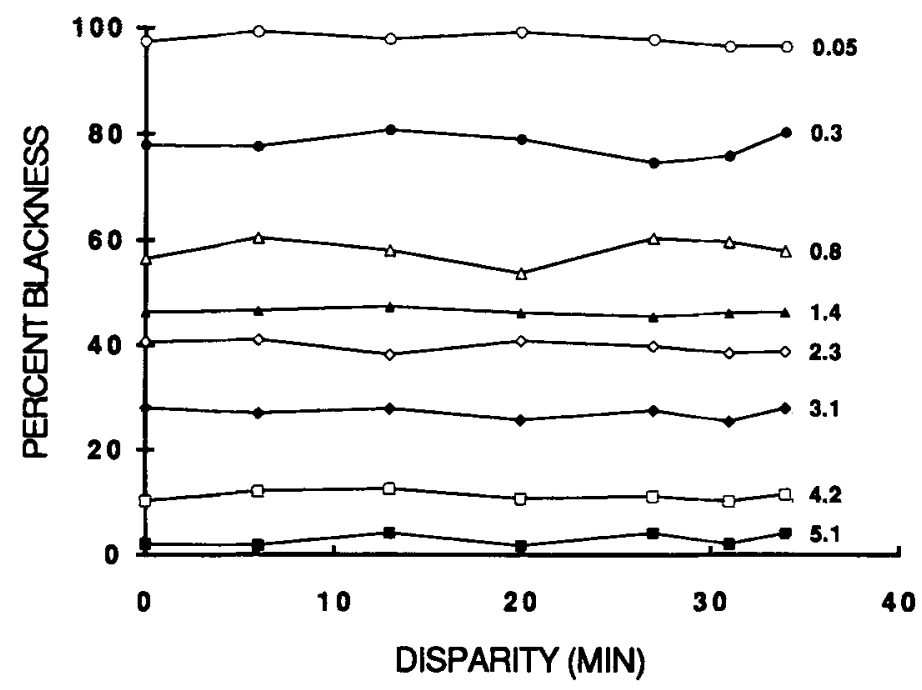

Figure 6. Percentage of blackness as a function of disparity in the hi/lo condition. Each function represents a different test luminance level (fL). The data are averages for all seven subjects.

The mean lightness responses of all of the subjects for the control stimuli (Types 1 and 2) across all contrast ratios produced functions resembling those shown in Figure 2 . The results implied that the subjects in Experiment 2 perceived lightness similarly to the way those in Experiment 1 perceived it. Furthermore, like the control for Experiment 1, the results confirmed that the lateral positioning of the fields during the study had no impact on the subjects' lightness perception.

Figure 6 displays the subjects' responses regarding the percentage of blackness for each disparity level of the hi/lo condition in the main experiment. Each of the 8 functions represents a different luminance level of the test field in fL and also, therefore, 2 different contrast ratios between the test and the inducing fields. Note that the functions are essentially linear with zero slope, indicating that there is no substantial change in lightness perception across disparity levels; that is, regardless of the inducing field with which the test is coplanar-or even if it is coplanar with neither inducer-there is no notable lightness shift. Figure 7 shows subjects' responses for the lo/hi condition. Here, too, the functions are essentially linear with zero slope and show no consistent lightness shift.

In Figure 8, the results of the three-field experiment and the two-field experiment in the hi/lo condition are

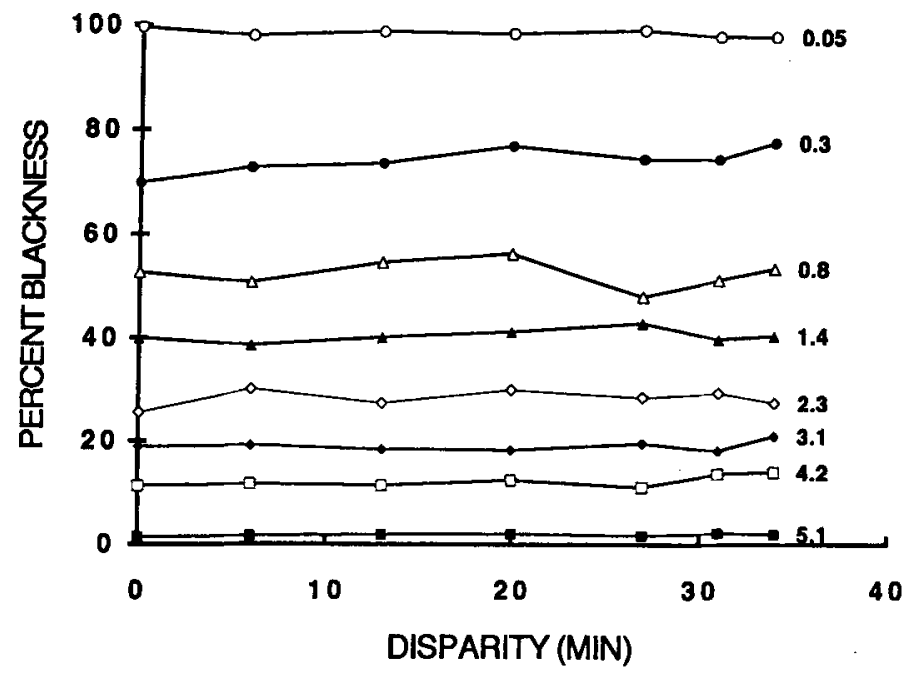

Figure 7. Percentage of blackness as a function of disparity in the lo/hi condition. Each function represents a different test luminance level (fL). The data are averages for all seven subjects. 
compared. The filled squares plot the slopes ( $y$ axis) derived from Figure 6 relating perceived blackness and disparity to the test-field luminance ( $x$ axis). The fact that they are small $( \pm .05)$ merely confirms the impression given by Figure 6 that the lightness of the test region is independent of its perceived depth with respect to the near and far inducers. The filled triangles in Figure 8 represent the slopes ( $y$ axis) derived from the two-field experiments relating perceived blackness to the testfield luminance ( $x$ axis). Each data point represents the slope of the line between the lightness response for a test field isolated against the far inducer relative to its isolated appearance against the near inducer. The derivation of the slopes assumes that if the coplanar hypothesis were true, the test region in the two-field experiment, when adjacent to and actually coplanar with just the far inducer (or just the near inducer), should appear identical in lightness to the test region in the three-field condition when the latter test region is perceptually coplanar with the far inducer at zero disparity (or, correspondingly, with the near inducer at $34^{\prime}$ of disparity). In other words, if the coplanar hypothesis were true, in the three-field experiment, the perceptually non-coplanar inducing field should make no contribution to the lightness of the test region; thus, in Figure 8, the filled squares should follow the filled triangles, which clearly they do not. The slopes are similar only for the lowest and highest test-field luminances. This would be expected regardless of whether the coplanar hypothesis were true because at the lowest test luminance, the stimulus appears to be nearly $100 \%$ black for both values of the inducers, and at the highest test luminance, the stimulus appears to be nearly $100 \%$ white ( $0 \%$ black) for both values of the in- ducers. These are what might be called basement and ceiling effects. For all the other values of the test luminance (i.e., those more or less in the middle of the graph area), the three-field and two-field results are radically different. The three-field results show that the lightness of the test is determined by both inducers and is independent of the perceived depth plane. The two-field experiments serve as a kind of baseline and merely reveal the expected result that lightness is determined by the contrast ratio (i.e., luminance of test divided by luminance of inducer). Although somewhat less convincingly, Figure 9 shows the same pattern of results for the lo/hi condition. The failure of the coplanar hypothesis can also be demonstrated by comparing selected blackness judgments in the two- and three-field experiments, which has the advantage of revealing how badly the hypothesis fails in terms of what people see-or at least in terms of what they say they see.

Figures $10,11,12$, and 13 plot the responses regarding percentage of blackness for the two-field control experiment against the responses for the three-field main experiment at each test field luminance level. Figure 10 displays the results of the hi/lo stimulus arrangement at $34^{\prime}$ of disparity. In the two-field experiment, the test region appears in the same plane as the near, lowluminance induction field at $34^{\prime}$ of disparity, while in the three-field experiment, the test is similarly situated, but the far, high-luminance induction field also appears in the display. According to the coplanar ratio hypothesis, the lightness judgments for each experiment at each luminance level should correlate in a one-to-one manner, because the test field is coplanar with the near inducer in both displays. In Figure 10, then, if this hy-

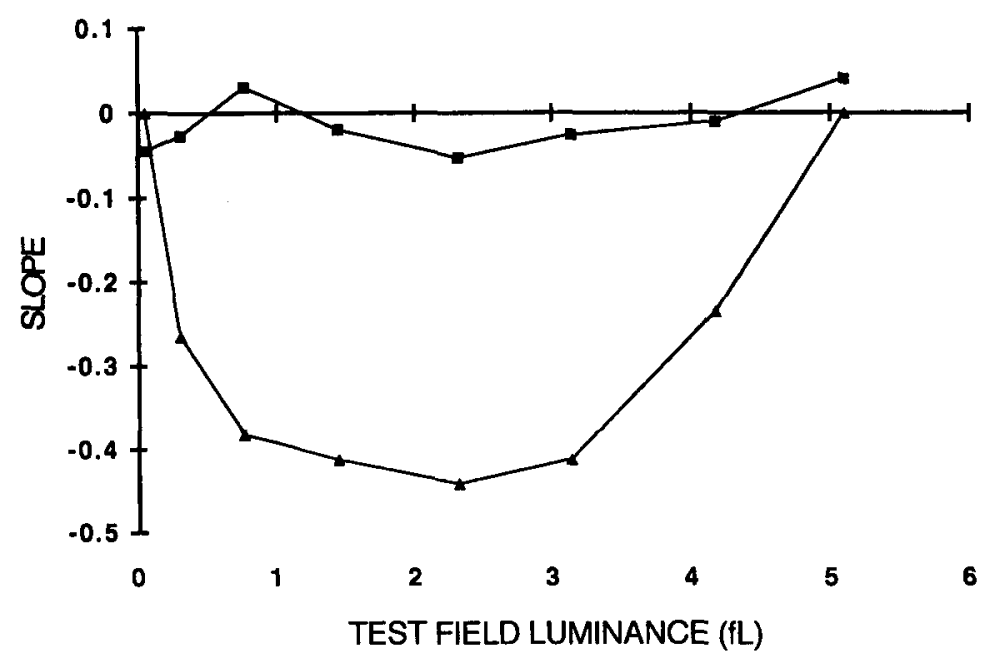

Figure 8. Slopes of the lines formed between the $0^{\prime}$ and $34^{\prime}$ disparity lightness responses for the hi/lo three-field experiment as a function of test-field luminance (squares). Also shown are the slopes between the hi/lo two-field lightness responses, when the test appeared isolated against the far inducer, and when it appeared isolated against the near inducer (both with no apparent depth) as a function of test-field luminance (triangles). 


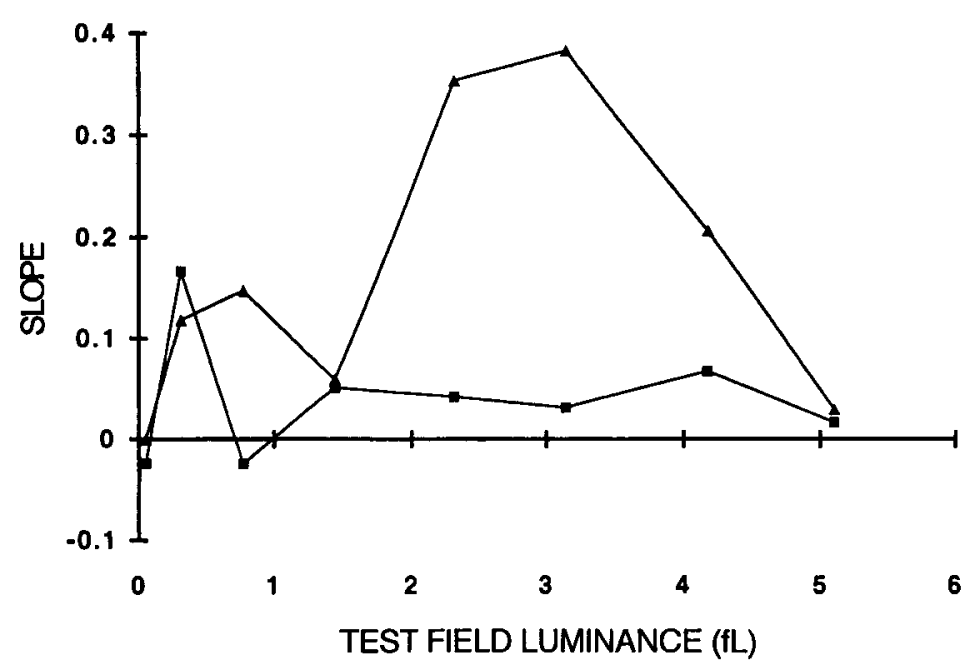

Figure 9. Slopes of the lines formed between the $0^{\prime}$ and $34^{\prime}$ disparity lightness responses for the lo/hi three-field experiment as a function of test-field luminance (squares). Also shown are the slopes between the lo/hi two-field lightness responses, when the test appeared isolated against the far inducer, and when it appeared isolated against the near inducer (both with no apparent depth) as a function of test-field luminance (triangles).

pothesis were true, we should expect the function to have a slope equal to one. Although a line with a slope of one appears on the graph, it is clear that our function systematically deviates from this line. These results thus show the coplanar ratio hypothesis to fail in a situation about which it makes very explicit predictions. It appears that in the three-field experiment, the far, noncoplanar induction field affected lightness judgments. For example, a stimulus that resulted in a $58 \%$ blackness rating in the three-field condition resulted in only a $38 \%$ blackness rating in the two-field condition.

In Figure 11, as in Figure 10, the lightness responses presented are those for the hi/lo condition, but in this case the test field appears to be coplanar with the far, high-luminance induction field (at $0^{\prime}$ of disparity). According to the predictions of the coplanar ratio hypothesis, when the test field is coplanar with the far inducer in both the two- and the three-field experiments, the lightness responses should match. Therefore, the responses should sketch a line with a slope equal to one (as appears in Figure 11). The actual function, however, deviates considerably from this line, making it clear that the lightness judgments for the test field are very different for each of the two experiments. It is apparent that the presence of two induction fields in the main experiment greatly alters the function from the one-to-one relationship anticipated by Gilchrist's $(1977,1980)$ theory.

Figure 12 shows a plot for the $34^{\prime}$ disparity condition of the lo/hi stimulus arrangement. In this display, the far induction field is of low luminance, while the near induction field is highly luminous. The test field appears in the same plane as the near induction field. These results, again, are notably different from the one-to-one relationship predicted by the coplanar ratio hypothesis.
Finally, Figure 13 shows the same lo/hi condition, but this time the test field is coplanar with the far induction field (at $0^{\prime}$ of disparity). Again, the one-to-one correspondence between lightness judgments predicted by the coplanar ratio hypothesis does not exist here.

Note that in Figures 10-13, in each case the data depart from the slope of the line predicted by the coplanar hypothesis. Although the values of $0 \%$ and $100 \%$ blackness are more nearly identical in both functions, at these extreme values, there tended to be basement and ceiling

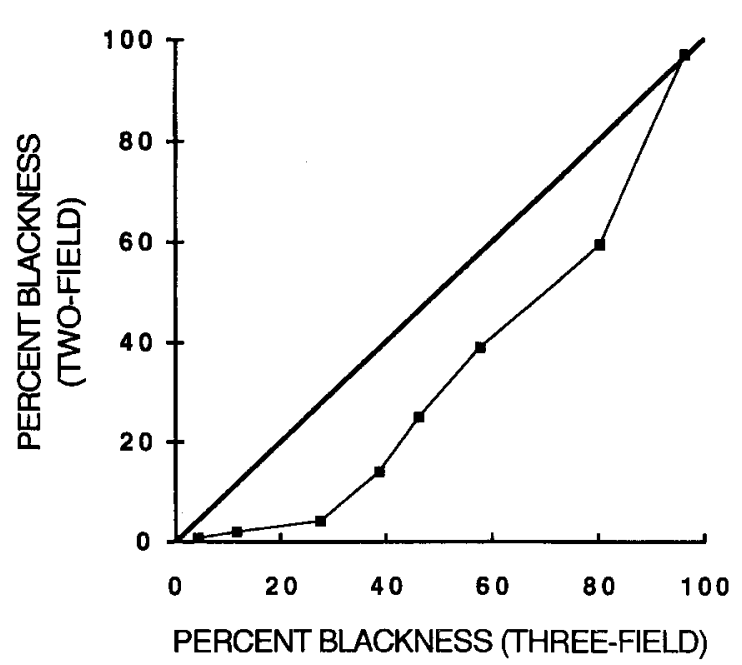

Figure 10. Percentage of blackness reported in the two-field experiment plotted against that reported in the three-field experiment. The data are for the hi/lo stimulus arrangement at $34^{\prime}$ of disparity, where the test field appeared coplanar with the near, low-luminance induction field. 
effects regarding lightness judgments. This was not, however, the case for the midrange values, where the test field was influenced by both induction fields present.

Experiment 2 clearly shows that in a simple viewing situation, with a single test field and two induction fields, perceived lightness does not change as a function of coplanarity, but is, in fact, constant. Our results explicitly contradict the predictions of Gilchrist's (1977, 1980) coplanar ratio hypothesis. As the test field moved from the plane of one induction field to that of another, the subjects' responses regarding percentage of blackness remained reliably uniform at each luminance level.

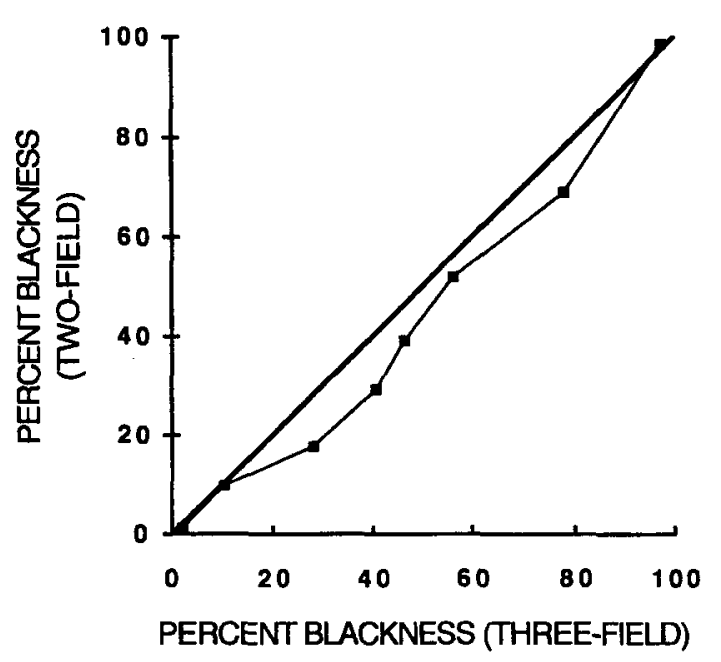

Figure 11. Percentage of blackness reported in the two-field experiment plotted against that reported in the three-field experiment. The data are for the hi/lo stimulus arrangement at zero disparity, where the test field appeared coplanar with the far, high-luminance induction field.

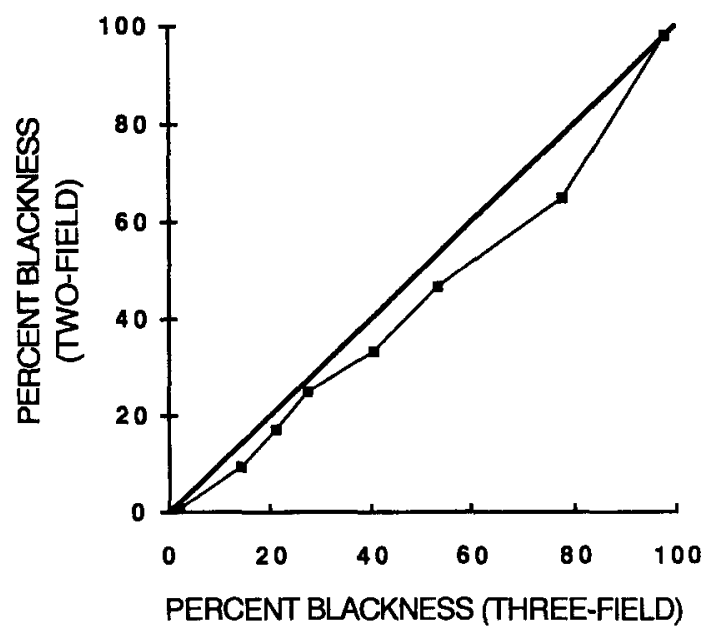

Figure 12. Percentage of blackness reported in the two-field experiment plotted against that reported in the three-field experiment. The data are for the lo/hi stimulus arrangement at $34^{\prime}$ of disparity, where the test field appeared coplanar with the near, high-luminance induction field.

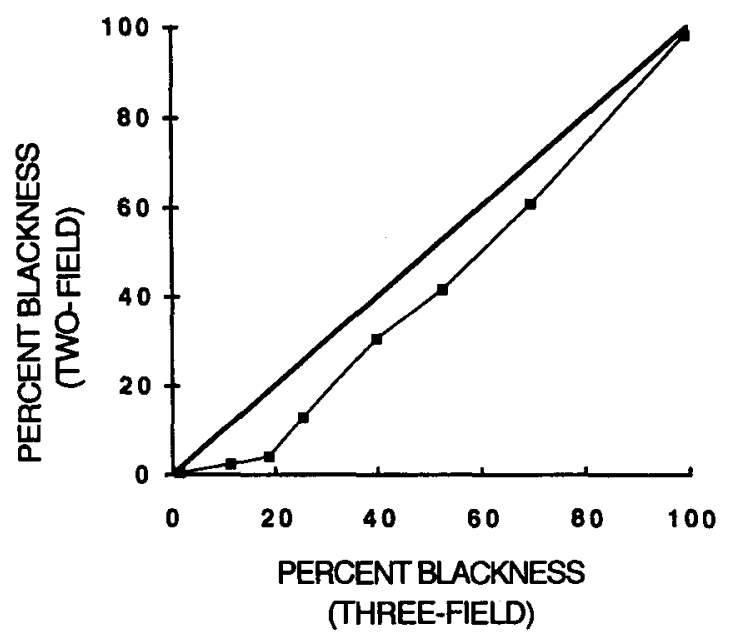

Figure 13. Percentage of blackness reported in the two-field experiment plotted against that reported in the three-field experiment. The data are for the lo/hi stimulus arrangement at zero disparity, where the test field appeared coplanar with the far, low-luminance induction field.

We thus conclude that the test field is influenced by both induction fields, regardless of their positions in depth.

\section{GENERAL DISCUSSION}

The results of Experiment 1 strongly suggested that lightness is invariant of perceived depth in a simple viewing situation. Experiment 2, however, clearly shows this to be true; despite the predictions of the coplanar ratio hypothesis, perceived lightness remained constant for a situation involving a single test field and two induction fields.

Given the findings of Experiments 1 and 2, previous research that found depth/lightness interactions must be reexamined.

\section{Gelb Effect Studies}

The large difference between the results of Experiment 1 and those of Gogel and Mershon (1969) might be attributable to the latters' use of the Gelb effect, which was unlike any of the contrast displays that we or other researchers used. Although evidence exists that the Gelb effect is explained by contrast mechanisms (Stewart, 1959), there is also support for the idea that cognitive factors such as attention influence the effect (Brussell \& Festinger, 1973), and the latter could have played a role in the studies by Gogel and Mershon (1969; Mershon \& Gogel, 1970). Gogel and Mershon (1969) found that over repeated presentations of the Gelb disk and induction object, the Gelb effect decreased in magnitude by $38 \%$ from the first to the third presentation. In their later study (Mershon \& Gogel, 1970), they reported an order effect during presentations in the Gelb paradigm. Both findings suggest that their subjects were no longer naive concerning the Gelb effect, and that some cognitive variable had intruded. 
Furthermore, in certain cases, Mershon and Gogel (1970) were unable to find reliable effects during repeated trials. Some of their results showed no significant difference in terms of perceived lightness between stereoscopic and monocular presentations of a Gelb disk and an induction field separated in depth (Table II, p. 62). They attributed this result to the fact that, on average, the degree of depth perceived in each condition was about the same, and claimed that this supported their hypothesis that it is the degree of depth perceived, and not the degree of the depth cue present, per se, that results in lightness shifts. However, these results conflict with findings reported earlier in this same study, where an apparent shift in lightness did occur between these same conditions. The unreliable nature of the depth and lightness percepts experienced during repeated trials in Mershon and Gogel's study emphasizes the complexities of the Gelb-effect paradigm and the difficulties involved in interpreting results obtained by its use.

Gogel and Mershon (1969, 1977; Mershon \& Gogel, 1970) emphasize that the variable that affects lightness is perceived depth, and not merely the extent of the depth cue used. In Experiment 1, of the present study, however, at least the same degree of perceived depth was attained $(23.20 \mathrm{~cm}$; see Figure 3$)$ as was reported by Mershon and Gogel (1970), and no depth/lightness interaction occurred. Mershon and Gogel also repeated that the magnitude of lightness-induction effects decreased with repeated presentations. We, however, found no such phenomenon, even in our most labile conditions (i.e., Experiment $1,0.43$ contrast ratio, $0^{\prime}, \pm 60^{\prime}$ disparity). Again, the Gelb-effect paradigm involves a complex viewing situation in which results remain difficult to interpret.

\section{Multifield Studies}

Because perceived depth and changes in coplanarity did not alter lightness percepts for the simple display situations of Experiments 1 and 2, a reexamination of Mershon (1972) is necessary. Despite its complexities, Mershon's experiment shares similarities with both Experiments 1 and 2 . It is therefore useful to consider the lightness judgments both for a subset of the display resembling Experiment 1 and also for the entire display, since it is somewhat like Experiment 2. The subset involves the test field and one pair of induction fields. Mershon (1972) found that as the test field was moved out of the plane of one pair of induction fields (termed the near induction object), so that it appeared to be behind this pair, the experience of lightness was affected. In fact, for the most extreme "behind" condition, we calculate that the obtained lightness shift corresponds in our terms to a $12.75 \%$ blackness difference. Furthermore, when the display is analyzed as a whole, Mershon's obtained lightness shift corresponds to $5.38 \%$ blackness between the two extreme positions, in which the test region was coplanar with, but laterally displaced between, the near and the far induction objects. Both of these lightness shifts are puzzling, given the uniformity of lightness judgments obtained in our own studies.
Moreover, besides Mershon's (1972) apparent large differences in lightness percepts, several other aspects of his results remain perplexing. For instance, although a change in lightness was found for the "behind" situation, no significant change in lightness was found when the test appeared in front of the near induction fields. Further, Mershon never states the direction of the perceived shifts in lightness obtained. It is not known whether the test appeared lighter or darker to the subjects as it moved in depth.

Another important concern is Mershon's (1972) inability to find a significant difference in perceived lightness on the basis of contrast ratios. In analyzing the results, he collapsed the 2 contrast-ratio conditions ( 0.06 and 0.02 ) across each other because no perceived difference in lightness was obtained between them. These results seem inexplicable, though, since contrast is generally accepted to be at least the main source of the lightness experience. In fact, the results of Experiment 1 predict an average blackness difference of $6 \%$ between these 2 ratios, and found a reliable perceived difference in lightness between contrast conditions of 0.01 and 0.03 (see Figure 2).

Mershon's (1972) findings, so unlike ours, demand that we compare his display and ours. The major differences involve Mershon's use of the two pairs of induction fields, the gap that existed between each pair, and the individual isolation of all fields against the low-luminance background. The possible effects of the gap on lightness judgments are unknown. One unfortunate possibility is that, due to the gaps in the display, a Gelb effect may have occurred because the test and induction fields were always isolated against the low-luminance background. If this was the case, the potential problems inherent in a Gelb-effect paradigm (as outlined previously for the studies by Gogel \& Mershon [1969; Mershon \& Gogel, 1970]) may also have been introduced into Mershon's (1972) experiment. Such problems include the possible intrusion of cognitive variables, such as attention, and also the unreliability of effects over time, such as decreased lightness induction after multiple presentations.

Finally, we must ask why Gilchrist's $(1977,1980)$ coplanar ratio hypothesis failed for the simple viewing situations used in Experiments 1 and 2. The most important reason is probably that the hypothesis is derived from experiments using highly complex viewing arrangements. Gilchrist's subjects were shown a mini-room within a room, containing multiple regions of various sizes, shapes, and luminance values, and they were asked to determine the lightness of a specified test field. The more complex the display, however, the more difficult it is to separate potential multiple-field interactions from the study of a depth/lightness interaction. Furthermore, this natural viewing situation may have been affected by uncontrolled aspects of illumination, such as shadows, edge discontinuities, specular reflection, and other inhomogeneities. Also, as mentioned earlier, the subjects were never informed of the distinction between lightness 
and brightness, which could have affected their responses. Additionally, in his key experiment, Gilchrist $(1977,1980)$ used monocular viewing and interposition as the cue to depth, differing greatly from the stereopsis used in our studies. Any of these potential factors may have caused Gilchrist to find large shifts in perceived lightness, in contrast to our finding that lightness is invariant for a simple viewing situation. We do not question the validity of Gilchrist's results under his conditions; we do, however, question the generality of his conclusions.

It seems most likely that the coplanar hypothesis applies to viewing situations in which illumination is taken into account as an explicit factor. This conclusion is consistent with the following remarks by Gilchrist (1980) concerning informal comments about the task made by the subjects.

First, the observers reported that the two tabs appeared to have similar intensities even though one appeared black and one appeared white. For example, one observer commented that the tabs would have the same intensity in a photograph of the display. Second, observers reported that the horizontal surfaces appeared more brightly illuminated than the vertical surfaces. (p. 532)

Thus, his subjects seem to have been performing some kind of task in which illumination was an important factor.

We reach a similar conclusion concerning a recent attempt to replicate Gilchrist's $(1977,1980)$ work by Schirillo et al. (1990). In order to avoid illumination cues, they simulated Gilchrist's display using a computer screen. They also attempted to distinguish between brightness and lightness. It may be important that they, like us, used stereoscopic cues rather than simply interposition. In the results of their brightness task, they found no effect related to depth. Their lightness task, however, gave results that were similar to those of Gilchrist. Thus, they concluded that the coplanar hypothesis was confirmed for lightness, but not for brightness. We, however, tentatively reach a different conclusion following an examination of Schirillo et al.'s instructions to their subjects, the relevant part of which is as follows:

Brightness match. Look over the 15 patches at the bottom of the display and select one that has the same brightness as the designated patch in the upper display. Disregard, as much as possible, the other areas of the upper display.

Lightness match. Look over the 15 patches at the bottom of the display and select one that looks as if it were cut from the same piece of paper as the upper display. Utilize, as much as possible, the other areas of both displays in making your comparison.

We feel that Schirillo et al.'s (1990) "brightness match" is most likely a lightness judgment, since it involves simulated surface colors. As such, it would be equivalent to our black/white (i.e., lightness) judgments. Their "lightness match" is apparently an explicit cognitive task that amounts to inferences about actual physical reflectance (i.e., "cut from the same piece of paper"). Such a task would, of course, involve some estimation about the illumination, which is where examining the entire display may enter into the picture. This latter task, we suggest, is similar to that performed by Gilchrist's $(1977,1980)$ subjects-hence the similarity of the results. In conclusion, we feel that Gilchrist's results and Schirillo et al.'s (1990) "lightness" results could only be obtained using a cognitive task with either real or simulated illumination factors.

In conclusion, Experiments 1 and 2 of the present study sought the entire stimulus space of multiplanar, simple viewing situations for indications of a depth/ lightness interaction. Our results show that when stereopsis and contrast levels are the only available cues, depth and lightness percepts are independent, and that it is retinal ratios, not coplanar ratios, that dictate lightness perception. It therefore appears that lightness determination is a perceptual function of a lower order than depth determination. Neural encoding of lightness likely occurs before the site of binocular interaction because lightness was found to remain unaffected by stereoscopic depth. We can conclude that before the relative depth location of an object is determined, its lightness value is known through sensory-level processes.

\section{REFERENCES}

Brussell, E. M., \& Festinger, L. (1973). The Gelb effect: Brightness contrast plus attention. American Journal of Psychology, 86, 225235.

GiBBs, T., \& LaWson, R. B. (1974). Simultaneous brightness contrast in stereoscopic space. Vision Research, 14, 983-987.

Gilchrist, A. L. (1977). Perceived lightness depends on perceived spatial arrangement. Science, 195, 185-187.

GILChrist, A. L. (1980), When does perceived lightness depend on perceived spatial arrangement? Perception \& Psychophysics, 28, 527-538.

Gogel, W. C., \& Mershon, D. H. (1969). Depth adjacency in simultaneous contrast. Perception \& Psychophysics, 5, 13-17.

GoGEL, W. C., \& MERshoN, D. H. (1977). Local autonomy in visual space. Scandinavian Journal of Psychology, 18, 237-250.

HeinemanN, E. G. (1955). Simultaneous brightness induction as a function of inducing- and test-field luminances. Journal of Experimental Psychology, 50, 89-96.

HERING, E. (1964). Outlines of a theory of light sense (L. M. Hurvich \& D. Jameson, Trans.). Cambridge, MA: Harvard University Press. (Original work published 1920)

JAMESON, D., \& HuRvich, L. (1961). Complexities of perceived brightness. Science, 133, 174-178.

JuLEsz, B. (1971). Foundations of cyclopean perception. Chicago: University of Chicago Press.

LIE, I. (1969). Psychophysical invariants of achromatic colour vision: IV. Depth adjacency and simultaneous contrast. Scandinavian Journal of Psychology, 10, 282-286.

Mershon, D. H. (1971). Evidence for the relationship of depth adjacency to contrast. A response to Lie. Scandinavian Journal of Psychology, 12, 295-302.

MershoN, D. H. (1972). Relative contributions of depth and directional adjacency to simultaneous whiteness contrast. Vision Research, 12, 969-979.

Mershon, D. H., \& Gogel, W. C. (1970). Effect of stereoscopic cues on perceived whiteness. American Journal of Psychology, 83, 55-67.

Quinn, P. C., Wooten, B. R., \& Ludman, E. J. (1985). Achromatic color categories. Perception \& Psychophysics, 37, 198-204.

Rock, I. (1983). The logic of perception. Cambridge, MA: MIT Press. Schirillo, J., Reeves, A., \& Arend, L. (1990). Perceived lightness, but not brightness, of achromatic surfaces depends on perceived depth information. Perception \& Psychophysics, 48, 82-90. 
SEWALl, L. (1990). Determinants of achromatic constancy. Unpublished doctoral dissertation, Brown University, Providence, RI.

Sewall, L., \& Wooten, B. R. (1991). Stimulus determinants of achromatic constancy. Journal of the Optical Society of America $A, \mathbf{8}$. 1794-1809.

STEWART, E. C. (1959). The Gelb effect. Journal of Experimental Psychologi, 57, 235-242.

von Helmholtz, H. (1925). Treatise on physiological optics (Vol. 3 , 3rd German ed.: J. P. C. Southall, Ed. and Trans.). Rochester, NY: Optical Society of America. (Original work published 1867)

Wal.LACH, H. (1948). Brightness constancy and the nature of achromatic colors. Journal of Experimental Psvchology, 38, 310-324.

\section{NOTES}

1. Certain early researchers considered achromatic colors (hues ranging from black, through gray, to white) as described by a single dimension known as brightness. Heinemann (1955) and Jameson and Hurvich (1961) approached the study of achromatic color using this single dimension. Others, however, favored a bidimensional view of achromatic colors, the two dimensions considered being brightness and lightness (see Sewall \& Wooten, 1991, for a review). Brightness refers to the perceived intensity of the light reflecting from an achromatic object. and ranges from dim to bright. Lightness, on the other hand, refers to the perceived achromatic color, from black, through gray, to white.

2. The Gelb effect is produced when a light source shines on a large disk of low reflectance. When the light source shines only on this disk and onto no other adjacent regions, the disk will appear white in color. However, when a smaller disk of high reflectance is presented against the large disk, the latter will suddenly appear black or dark gray.

3. A contrast ratio is defined as the luminance of the test field divided by the luminance of the induction field, such that the lower the luminance of the test, the smaller the ratio.

(Manuscript received July 6, 1992; revision accepted for publication September 26, 1994.) 